\title{
Elastic and Elastic-Plastic Behaviour of a Crack in a Residual Stress Field
}

\author{
Guiyi Wu ${ }^{a}$, Chris Aird ${ }^{b}$, David Smith and Martyn Pavier ${ }^{c^{*}}$ \\ Department of Mechanical Engineering, University of Bristol \\ Queen's Building, University Walk, Bristol BS8 1TR, UK \\ aguiyiwu@outlook.com, bchris.aird@edf-energy.com, martyn.pavier@bristol.ac.uk
}

\begin{abstract}
Keywords: Fracture Mechanics, Residual Stress, Finite Element Analysis, Strip Yield Model, Plasticity
\end{abstract}

\begin{abstract}
The behaviour of a crack in the centre of a plate subject to a far-field stress has been studied where the plate contains an initial residual stress. Elastic and elastic-plastic conditions have been considered. For elastic conditions a series of analyses based on stress intensity factor solutions have been developed to calculate the state of opening and the stress intensity factor for cracks of different lengths relative to the size of the residual stress field and different magnitudes of applied stress relative to the magnitude of the residual stress. For elastic-plastic conditions a strip yield model has been used to develop a similar set of analyses. The results of these analyses compare closely with those of finite element modelling.
\end{abstract}

\section{Introduction}

In an engineering component containing a crack, residual stresses interact with stresses generated by applied loading in a complex manner. Typically, numerical techniques are required to predict the likelihood of fracture, providing results that are particular to the set of conditions considered. The work we describe here represents an attempt to understand the generic behaviour of the crack for a straightforward geometry, loading and residual stress distribution that we hope will provide insight into the results of more involved analyses.

Terada [1] provided an early analysis of the effect of residual stress on a crack. He proposed a residual stress distribution to represent a butt weld between two plates and then developed a method to evaluate the stress intensity factor assuming elastic behaviour. Chell and Ewing [2] discussed the effect of plasticity on fracture when residual stresses exist while Labeas and Diamantakos [3] used finite element analysis to explore the stress intensity factor for a crack of varying length embedded in a residual stress field.

In this paper we investigate the behaviour of a crack in an infinite two dimensional plate with an initial residual stress distribution under superimposed uniaxial tension. First we describe our method for generating a residual stress distribution using a stress function. Next we use existing stress intensity solutions to explore the linear elastic behaviour of the crack as the length of the crack is altered and the magnitude of the applied stress is increased. We then use the strip yield model to investigate the elastic-plastic behaviour of the crack with increasing applied stress, for one size of the crack.

\section{Residual Stress}

For the analytical work we a residual stress distribution chosen as representative of the residual stresses produced by a butt weld in a plate, as shown in Fig. 1. 


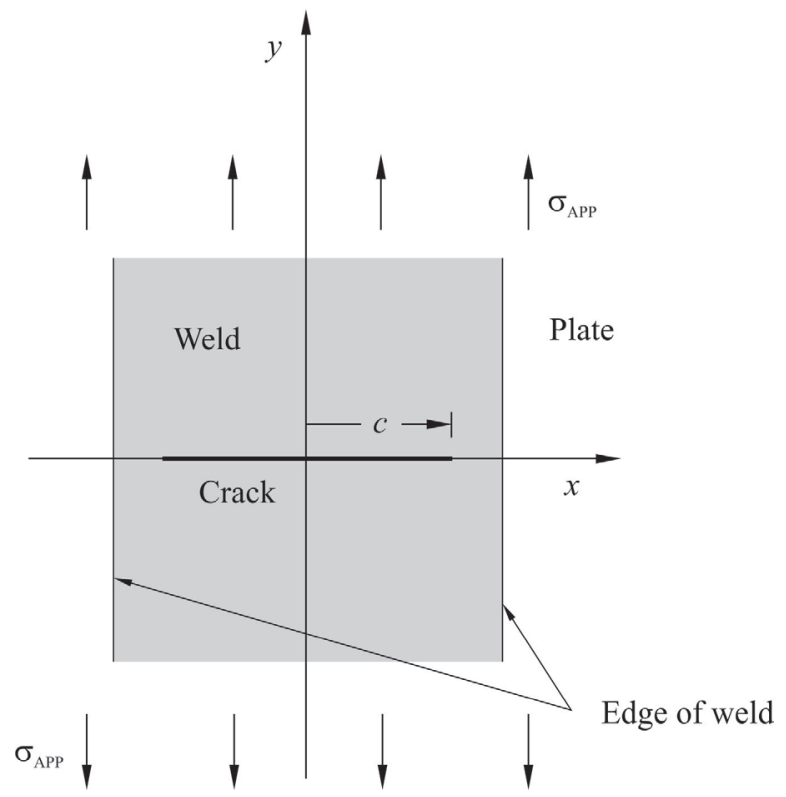

Fig. 1 Geometry of model.

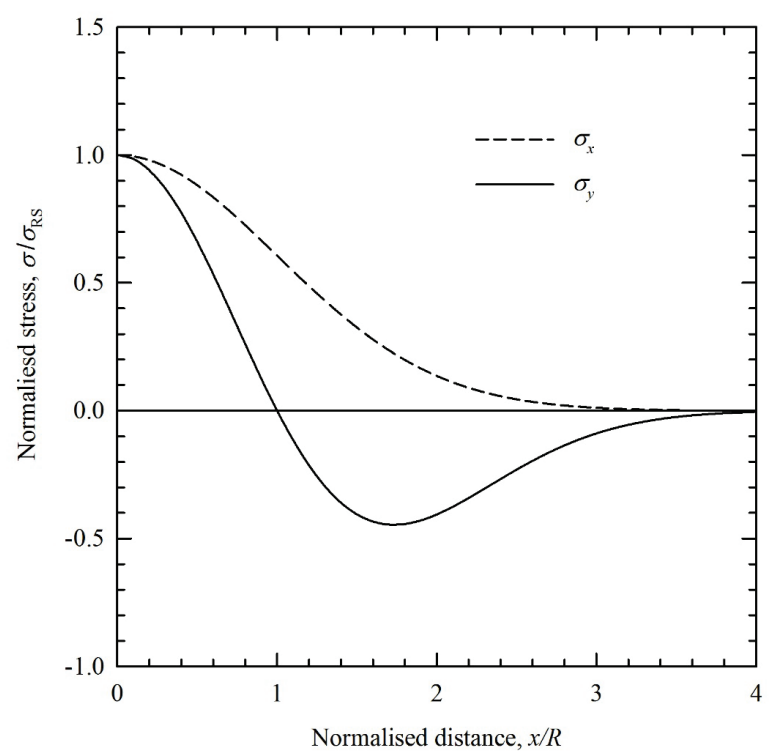

Fig. 2 Normalised residual stress

components $\sigma_{x x}$ and $\sigma_{y y}$ versus $x$ at $y=0$.

Our analytical results will be compared with those of finite element modelling using ABAQUS 6.11 , for which a residual stress distribution in equilibrium must be generated. This is achieved by defining the stress function

$$
\phi=-\sigma_{\mathrm{RS}} R^{2} \exp \left[-\frac{x^{2}}{2 R^{2}}-\frac{y^{2}}{2 R^{2}}\right]
$$

where $R$ characterises the size of the residual stress distribution and will be of the order of half the width of the wled in Fig. 1. Stress components are calculated from the stress function by

$$
\sigma_{x x}=\frac{\partial^{2} \phi}{\partial y^{2}}, \sigma_{y y}=\frac{\partial^{2} \phi}{\partial x^{2}}, \sigma_{x y}=\frac{\partial^{2} \phi}{\partial x \partial y}
$$

For $y=0$, this gives the same distribution for $\sigma_{y y}$ as proposed by Terada [1]:

$$
\sigma_{y y}=\sigma_{\mathrm{RS}}\left[1-\frac{x^{2}}{R^{2}}\right] \exp \left[-\frac{x^{2}}{2 R^{2}}\right]
$$

These stress components were used to input an initial stress distribution using the SIGINI routine. The graph of Fig. 2 plots the normalised stresses $\sigma_{x x}$ and $\sigma_{y y}$ versus $x$ for $y=0$.

\section{Elastic behaviour}

In this section the elastic behaviour is studied for a crack in a residual stress field subjected to additional uniaxial applied load in the $y$ direction as defined in Fig. 1. Depending on the length of the crack, the level of applied load and the magnitude of the residual stresses the crack may be closed, partially open or fully open.

Fig. 3(a) shows the behaviour of the crack for positive $\sigma_{\mathrm{RS}}$, that is when the residual stress is tensile at the centre. The half-length of the crack $c$ is normalised with respect to the size of the tensile region of the residual stress field $R$ and the applied stress $\sigma_{\text {APP }}$ is normalised with respect to $\sigma_{\mathrm{RS}}$. 
For all sizes of crack, the crack is always closed if the applied stress is compressive and greater in magnitude than the value of the residual stress at $x=0$. As the magnitude of the compressive applied stress is reduced, the crack opens first at $x=0$ when the sum of the applied and residual stresses equal zero. The boundary between the fully closed and partially open regions of crack behaviour, the line $A B$, is given by $\sigma_{\mathrm{APP}}=-\sigma_{\mathrm{RS}}$.

Once the crack is partially open, increasing the magnitude of the applied stress will eventually cause the crack to open completely. The magnitude of applied stress to open the crack fully depends on the length of the crack. For crack half-lengths less than $\sqrt{5} R$ the boundary between the fully open and partially open regions is the line $A C$. This line is given by the condition that the sum of the stress intensity factor at the tip due to the applied stress $K_{\mathrm{APP}}$ and the stress intensity factor due to the residual stress $K_{\mathrm{RS}}$ is equal to zero. $K_{\mathrm{RS}}$ is derived by integration of the expression for the stress intensity factor due to pairs of splitting forces applied to the crack surface presented in Tada, Paris and Irwin [4].

For crack half-lengths greater than $\sqrt{5} R$, if the applied stress is high enough that the crack is fully open and the applied stress is then reduced the crack closes first at some point along the crack surface. The position of this point depends on the crack length and is calculated by integration of the expression for crack opening due to pairs of splitting forces applied to the crack surface [4]. The boundary between the fully open and partially open regions is the line $C D$. For crack half-lengths greater than $\sqrt{5} R$ a small regime of crack behaviour exists as shown in Fig. 3(a) where the crack is partially open: open at the tips and in the centre but closed between these points. This regime is bounded by the two lines $C D$ and $C E$

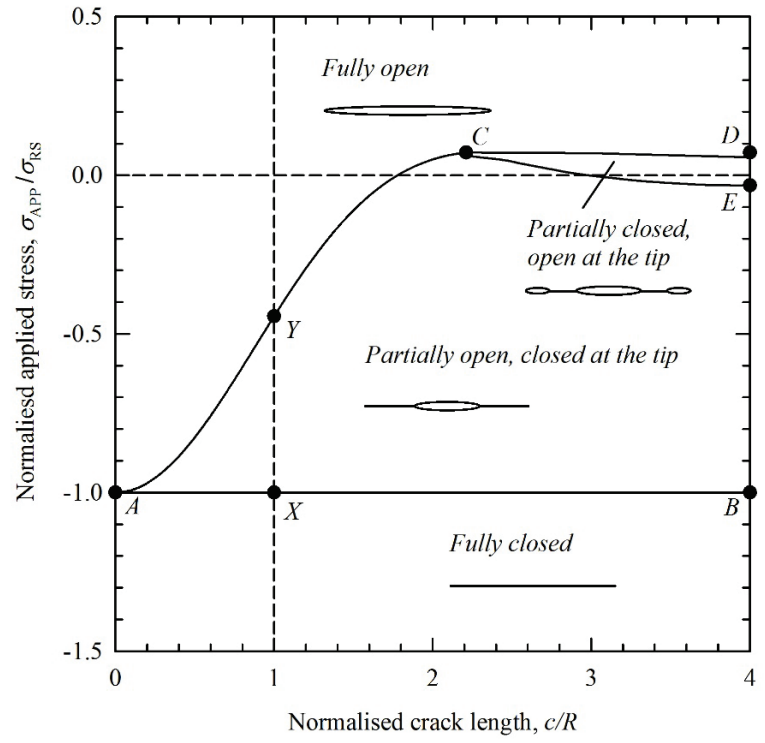

(a)

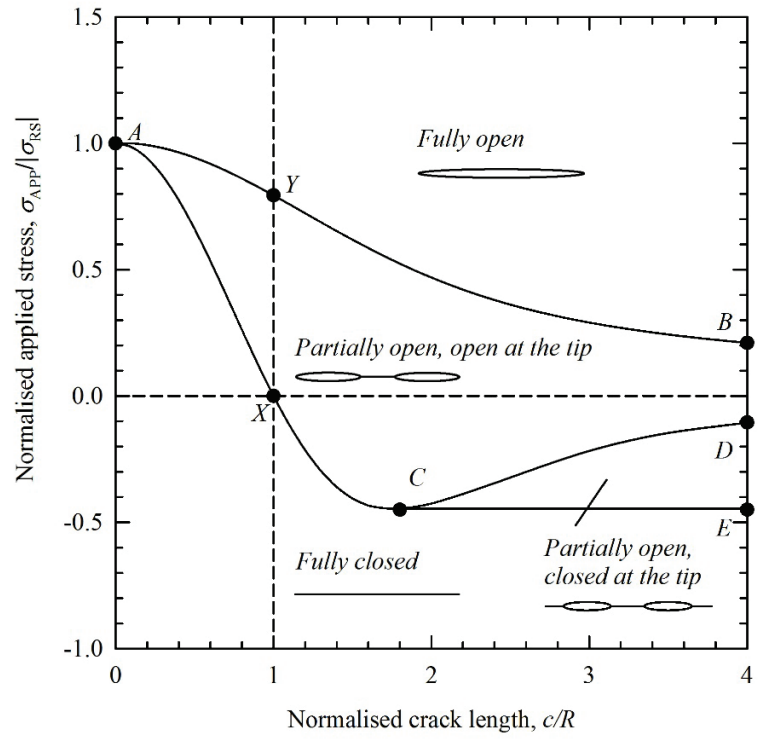

(b)

Fig. 3 Elastic crack behaviour map for (a) tensile residual stress and (b) compressive residual stress.

The behaviour of the crack for negative $\sigma_{\mathrm{RS}}$, that is when the residual stress is compressive at the centre, is shown in Fig. 3(b). The crack is now fully closed provided the applied load is less than a certain value that depends on the crack length. When the crack half-length is less than $\sqrt{3} R$ the crack opens first at the tip. Points on the line $A C$ are given by the condition that the sum of the residual 
stress and the applied stress equals zero at the crack tip. For crack half-lengths greater than $\sqrt{3} R$, the crack becomes open at the tip when the stress intensity at the tip, $x=c$, is zero. Points on the line $C D$ are given by simultaneous solution of the equations

$$
\begin{aligned}
& K_{\mathrm{APP}}^{a}+K_{\mathrm{RS}}^{a}=0 \\
& K_{\mathrm{APP}}^{c}+K_{\mathrm{RS}}^{c}=0
\end{aligned}
$$

$K_{\mathrm{APP}}^{a}$ and $K_{\mathrm{APP}}^{c}$ are the stress intensity factors for twin cracks with crack tips located at $x= \pm a$ and $x= \pm c$ due to a uniform applied stress [4].

For all sizes of crack, the crack is always fully open provided the applied stress is high enough. If the crack is fully open and the applied stress is then reduced the crack closes first at the centre. The applied stress at which closure occurs depends on the length of the crack. The boundary between the fully open and partially open regions is the line $A B$. This line is given by the condition that the sum of the opening at the centre of the crack due to applied stress $\delta_{\text {APP }}$ and the opening due to the residual stress $\delta_{\mathrm{RS}}$ is equal to zero.

In addition to the behaviour of the crack for different applied stresses, stress intensity factors may also be calculated. For example, Fig. 4 shows the stress intensity for a crack of half-length $c=R$ versus the applied stress for three cases: no residual stress, tensile and compressive residual stress. For the residual stress cases, these results correspond to the vertical dashed line in Fig. 3(a) and (b) with $c / R=1$.

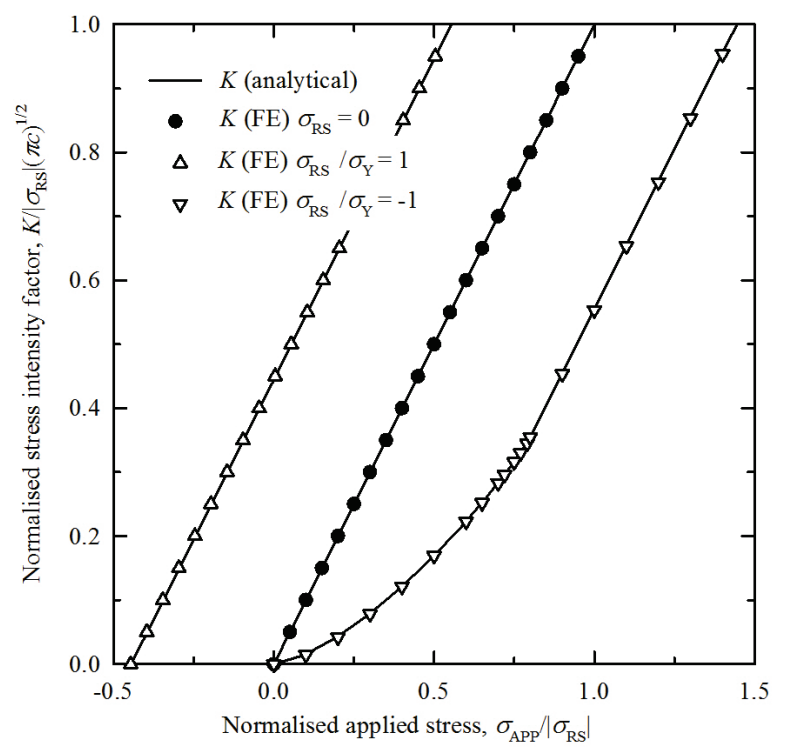

Fig. 4 Elastic normalised stress intensity factor $K /\left|\sigma_{R S}\right| \sqrt{\pi c}$ versus normalised applied stress $\sigma_{A P P} /\left|\sigma_{R S}\right|$ for zero, tensile and compressive residual stress.

The finite element results shown in Fig. 4 for comparison were evaluated using the ABAQUS 6.11 finite element system. Calculations of stress intensity factors used the JEDI code [5].

\section{Elastic-plastic behaviour}

We now use the strip yield model of Dugdale [6] and Barenblatt [7] to examine the elastic-plastic behaviour of a crack in a residual stress field. We will not present revised crack behaviour maps since in general these maps are similar to the maps for elastic behaviour of Fig. 3. Instead we choose one crack length defined by $c=R$ and study the behaviour of the crack as the applied load is increased from an initial state where the crack is fully closed. 
Whne the applied load is sufficiently high that the crack opens at the tip, a yielded zone of length $\rho$ forms ahead of the tip. The size of this yielded zone is calculated using the condition that the total stress intensity factor for an extended crack of half-length $c+\rho$ is zero. When residual stresses act in addition to the applied stress, the total stress intensity factor $K_{\text {тот }}$ is calculated as the sum of the stress intensity factors due to the applies stress $K_{\mathrm{APP}}$, the residual stress $K_{\mathrm{RS}}$ and stresses applied to the crack tip to represent the yield zone $K_{\mathrm{Y}}$. Therefore

$$
K_{\mathrm{TOT}}=K_{\mathrm{APP}}+K_{\mathrm{RS}}+K_{\mathrm{Y}}=0
$$

Solution of Eq. (5) gives the size of the plastic zone $\rho$. The effective stress intensity factor is evaluated using the technique of Burdekin and Stone [8], which requires the crack opening displacement to be calculated at $x=c$. The total crack opening displacement $\delta_{\text {тот }}$ is evaluated as the sum of the crack opening due to the applied stress $\delta_{\mathrm{APP}}$, the residual stress $\delta_{\mathrm{RS}}$ and the stresses in the yielded zone $\delta_{\mathrm{Y}}$. That is

$$
\delta_{\mathrm{TOT}}=\delta_{\mathrm{APP}}+\delta_{\mathrm{RS}}+\delta_{\mathrm{Y}}
$$

Finally the effective stress intensity factor is obtained by

$$
K_{\mathrm{EFF}}=\sqrt{E^{\prime} \sigma_{\mathrm{Y}} \delta_{\mathrm{TOT}}}
$$

Fig. 5 compares the results of the strip yield model for tensile residual stress and compressive residual stress, where the magnitude of the residual stress is equal to the yield stress, that is $\sigma_{\mathrm{Y}} /\left|\sigma_{\mathrm{RS}}\right|=1$. Fig. 5 also shows the results for the standard strip yield model, the case with no residual stress. The figure also shows two dimensional plane stress finite element results obtained for $K_{\mathrm{EFF}}$ using the calculation

$$
K_{\mathrm{EFF}}=\sqrt{E^{\prime} J}
$$

where $J$ is the value of the $J$-integral evaluated using the JEDI procedure [5]. Very good agreement is obtained even for applied stresses approaching the yield stress where large plastic zones develop and small scale yielding conditions certainly do not exist.

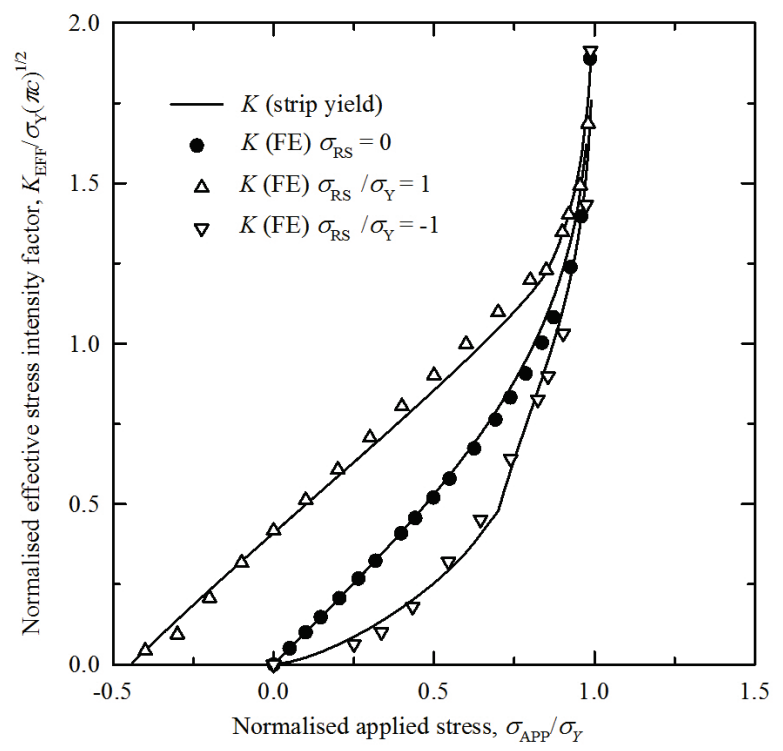

Fig. 5 Elastic-plastic normalised effective stress intensity factor $K_{\mathrm{EFF}} /\left|\sigma_{R S}\right| \sqrt{\pi c}$ versus normalised applied stress $\sigma_{A P P} /\left|\sigma_{R S}\right|$ for zero, tensile and compressive residual stress. 


\section{Conclusions}

Analytical and finite element methods have been used to map the elastic behaviour of a crack in a residual stress field. Depending on the length of the crack and the magnitude of the superimposed applied stress the crack may be fully open, partially open and open at the tip, partially open but closed at the tip or fully closed. Of course, a non-zero stress intensity factor only develops when the crack is open at the tip.

Effective stress intensity factors for an elastic-perfectly plastic crack in a residual stress field under plane stress conditions have been calculated using strip yield and finite element methods with good agreement, even for high levels of applied stress approaching the yield stress. The crack opening behaviour is similar to that for an elastic crack.

The results that have been presented were based on the distribution of residual stress of Eq. (3). Different distributions will give different results, but not markedly different since the calculations depend on a weighted average of the didtribution.

\section{References}

[1] Terada H. An analysis of the stress intensity factor of a crack perpendicular to the welding bead, Engng. Fracture Mech., 8 (1976), 441-111. http://dx.doi.org/10.1016/0013-7944(76)90024-2

[2] Chell GG and Ewing DJF. The role of thermal and residual stresses in linear elastic and post yield fracture mechanics. Int. J. Fracture, 13 (1977), 467-479

[3] Labeas G and Diamantakos I. Numerical investigation of through crack behaviour under welding residual stresses. Engineering Fracture Mechanics, 76 (2009), 1691-1702. http://dx.doi.org/10.1016/j.engfracmech.2009.03.006

[4] Tada H, Paris PC, Irwin GR (2000). The stress analysis of cracks handbook ( $3^{\text {rd }}$ edition), ASME Press, New York. http://dx.doi.org/10.1115/1.801535

[5] Beardsmore DW (2008). A code for the calculation of $\mathbf{J}$ for cracks inserted in initial strain fields for the role of $\mathrm{J}$ and $\mathrm{Q}$ in the predictions of crack extension and fracture. Proc. ASME 2008 Pressure Vessels and Piping Conference, Chicago, USA, Paper No. PVP2008-61169, 955-966. http://dx.doi.org/10.1115/pvp2008-61169

[6] Dugdale DS (1960). Yielding of steel sheets containing slits. J. Mech. Phys. Solids, 8, 100-104. http://dx.doi.org/10.1016/0022-5096(60)90013-2

[7] Barrenblatt DS (1962). The mathematical theory of equilibrium cracks in brittle fracture. Adv. Appl. Mech. 7, 55-125. http://dx.doi.org/10.1016/s0065-2156(08)70121-2

[8] Burdekin FM and Stone DEW (1966). The crack opening displacement approach to fracture mechanics in yielding materials, J. Strain Analysis for Eng. Design, 1, 145-153. http://dx.doi.org/10.1243/03093247V012145 